during diagnostic procedures (Dermatological Clinic) after informed consent and Ethical Committee approval. Cultured Fbs at 4th passage were treated with ET-1 $(100 \mathrm{nM})$, pretreated for $1 \mathrm{~h}$ with endothelin-A (2M) or endothelin-A/B receptors antagonist $(10 \mathrm{M})$ alone and follow by ET-1 for 24 and $48 \mathrm{~h}$ in RPMI1640 at $5 \%$ of fetal bovine serum. Untreated Fbs were used as controls (CNT). Polyclonal primary antibody against COL1 (dilution 1:100, Abcam, Cambridge, UK) and monoclonal primary antibody against FN (dilution 1:100, Sigma-Aldrich, Milan, Italy) were used to evaluate ECM protein synthesis by immunocytochemistry (ICC) and western blot analysis (WB). ICC image analysis and WB densitometry were performed by the Leica $\mathrm{Q}$-Win image analysis system. Experiments were done in triplicate. Statistical analysis was performed by Fridman test and $\mathrm{p}<0.05$ was considered significant.

Results ET-1 induced a significant increase in COL1 and FN synthesis both at $24 \mathrm{~h}$ ( $\mathrm{p}<0.001$ respectively) and $48 \mathrm{~h}$ $(p<0.001$ respectively) when compared to CNT (vs CNT). At $24 \mathrm{~h}$ endothelin-A receptor antagonist was able to decrease significantly COL1 and FN ( $p<0.001$, respectively) whereas at 48 h only COL1 synthesis was significantly reduced $(p<0.01)$ versus ET-1 treated Fbs. The endothelin-A/B receptors antagonist decreased significantly COL1 and FN synthesis both at 24 ( $p<0.01 ; p<0.001$ respectively) and $48 \mathrm{~h}(\mathrm{p}<0.05 ; \mathrm{p}<0.01$ respectively) versus ET-1 treated cells. WB analysis confirmed the results.

Conclusions The results confirmed the important role of ET-1 fibrosis in increasing the ECM protein synthesis, in particular COL1, which represents the major molecule over-expressed during this pathological process. The selective blockage of endothelin-A receptor seems more important in decreasing the COL1 synthesis whereas the endothelin-A/B receptors antagonist seem more efficiently in reducing the FN production. However, the treatment with the ET-1 receptors antagonists might be an important strategy to block the ET-1 effects on fibrotic process in SSc.

\section{A82 INFLUENCE OF ENDOTHELIN-1 RECEPTORS ANTAGONISTS ON EXTRACELLULAR MATRIX PROTEIN SYNTHESIS IN CULTURE OF HUMAN SCLERODERMA SKIN FIBROBLASTS}

Soldano S, ${ }^{1}$ Montagna P, Brizzolara R, ${ }_{1}^{1}$ Ferrone C, ${ }_{1}^{1}$ Parodi A, ${ }^{2}$ Cutolo $M^{1}{ }^{1}$ Research laboratories and Academic Unit of Clinical Rheumatology, Department of Internal Medicine, University of Genova, Genova, Italy; ${ }^{2}$ Department of Endocrinologial and Medical Science, Unit of Dermatology, University of Genova, Genova, Italy

\subsection{6/ard.2010.149104.26}

Background and objectives Skin fibrosis is an important pathological event in the systemic sclerosis (SSc) and is characterised by excessive synthesis and deposition of extracellular matrix (ECM) proteins by activated scleroderma fibroblasts (Fbs). ${ }^{1}$

Fbs activation is induced by different mechanisms including their stimulation by soluble mediator as endothelin-1 (ET-1). ${ }^{2} 3$ ET-1 is a vasoconstrictor able to induce the synthesis of profibrotic proteins. These effects are mediated by its receptors expressed on different cell types including Fbs. ${ }^{4}$

In this study both a selective endothelin- $A$ and an endothelin$A / B$ receptors antagonist were used. The aim of present study was to evaluate the effects of these antagonists on ECM protein synthesis in culture of SSc skin Fbs, in particular collagen type 1 (COL1) and fibronectin (FN).

Materials and methods Human Fbs were obtained by skin biopsies of six female SSc patients (mean age 68.55 years)

\section{REFERENCES}

1. Asano Y. Future treatments in systemic sclerosis. Dermatol. 2010;37:54-70.

2. Abraham DJ, Krieg T, Distler J, et al. Overview of pathogenesis of systemic sclerosis. Rheumatology 2009;48:iii3-iii7.

3. Chen Y, Shi-wen X, Eastwood M, Black CM, Denton CP, Leask A, Abraham DJ. Contribution of activin receptor-like kinase 5 (transforming growth factor beta receptor type I) signaling to the fibrotic phenotype of scleroderma fibroblasts. Arthritis Rheum. 2006;54:1309-16.

4. Sticherling $\mathbf{M}$. The role of endothelin in connective tissue diseases. Rheumatology 2006;45:iii8-iii10. 